01;05

\title{
Характеристики автоволн локализованной пластичности металлов и параметр Дебая
}

\author{
(ㄱ) Л.Б. Зуев, С.А. Баранникова \\ Институт физики прочности и материаловедения СО РАН, Томск, Россия \\ E-mail: Ibz@ispms.tsc.ru
}

Поступило в Редакцию 4 февраля 2019 г.

В окончательной редакции 4 фревраля 2019г.

Принято к публикации 16 апреля 2019г.

\begin{abstract}
Проанализирована зависимость характеристик автоволн локализованной пластичности от характеристического параметра Дебая (температуры Дебая) для девятнадцати различных металлов. Показано, что эта экспериментально установленная линейная зависимость является следствием ранее введенного упругопластического инварианта деформации, связывающего параметры упругих и пластических процессов в деформируемой среде.
\end{abstract}

Ключевые слова: деформация, пластичность, кристаллическая решетка, параметр Дебая.

DOI: 10.21883/PJTF.2019.14.48021.17725

Экспериментальное исследование кинетики развития макроскопически локализованной пластичности, проведенное для девятнадцати различных металлов $(\mathrm{Mg}, \mathrm{Al}$, Ti, V, $\alpha$-Fe, $\gamma$-Fe, Co, Ni, Cu, Zn, Zr, Nb, Mo, Cd, In, $\mathrm{Sn}, \mathrm{Hf}, \mathrm{Ta}, \mathrm{Pb})[1,2]$, показало, что на стадиях линейного деформационного упрочнения в них формируется фазовая автоволна локализованной пластичности. При этом удобной характеристикой процесса распространения такой автоволны является произведение ее длины $\lambda$ на постоянную для заданных условий нагружения скорость распространения $V_{a w}$. Обе эти характеристики с достаточной точностью определяются с помощью $X-t$-диаграмм [1] (рис. 1). Задачей настоящей работы является поиск связи автоволновых характеристик пластического течения с такой решеточной характеристикой материала, как параметр (температура) Дебая. Как оказалось, произведение $\lambda V_{a w}$ линейно растет с ростом параметра Дебая $T_{\mathrm{D}}$, нормированного на температуру $T^{*}$, определяемую как $T^{*}=E_{b} / k_{\mathrm{B}}$, где $k_{\mathrm{B}}-$ постоянная Больцмана. Энергия связи $E_{b}$ для исследованных элементов была взята из справочника [3]. Коэффициент корреляции величин $\lambda V_{a w}$ и $T_{\mathrm{D}} / T^{*}$ составляет $\sim 0.8$.

Исследуемая зависимость, показанная на рис. 2, описывается уравнением

$$
\lambda V_{a w} \approx \Lambda \frac{T_{\mathrm{D}}}{T^{*}}
$$

где коэффициент пропорциональности $\Lambda \approx 4.42 \cdot 10^{-5} \mathrm{~m}^{2} / \mathrm{s}$. Попытки использовать вместо энергии $E_{b}$ другие характерные для твердых тел значения энергии, например теплоту плавления, теплоту кипения или теплоту перехода пар-жидкость [3], показали существенно худшую корреляцию, коэффициент которой составил $0.59,0.75$ и 0.6 соответственно.

Как следует из данных рис. 2, соотношение (1) выполняется для металлов, относящихся к третьему, четвертому, пятому и шестому периодам Периодической системы элементов. В настоящей работе обсуждается смысл этого уравнения, причем анализ основан на ранее установленном [1] соотношении, согласно которому автоволновые и упругие характеристики деформируемой среды находятся в тесной взаимосвязи, уравнение которой имеет вид

$$
\lambda V_{a w}=\hat{Z} \chi V_{t}
$$

Здесь $\chi-$ межплоскостное расстояние, $V_{t}$ - скорость распространения поперечных акустических (упругих) волн, а $\hat{Z} \approx 1 / 2$. Соотношение (2) было проверено для разных материалов и разных видов деформирования и было названо упругопластическим инвариантом деформации [1,2]. Инвариант деформации имеет ряд следствий, которые описывают многие закономерности пластического течения [4]. Покажем, что это же относится и к уравнению (1).

Используя известные соотношения $k_{\mathrm{B}} T_{\mathrm{D}}=\hbar \omega_{\mathrm{D}} \quad$ и $V_{t}=2 \chi \omega_{\mathrm{D}}[5] \quad$ (где $\hbar=h / 2 \pi$ - постоянная Планка, $\omega_{\mathrm{D}}$ - дебаевская частота), а также вводя определенную выше температуру $T^{*}$, получаем из уравнения инварианта (2)

$$
\lambda V_{a w} \approx\left(2 \hat{Z} \chi^{2} \frac{k_{\mathrm{B}}}{\hbar} T^{*}\right) \frac{T_{\mathrm{D}}}{T^{*}}=\Lambda \frac{T_{\mathrm{D}}}{T^{*}} .
$$

Получившееся линейное по $T_{\mathrm{D}} / T^{*}$ уравнение (3), очевидно, также может считаться следствием из упругопластического инварианта деформации (2).

Рассчитанные по уравнению (4) значения коэффициента $\Lambda$ в уравнении (1)

\begin{tabular}{c|c|c|c|c|c|c|c}
\hline Параметр & $\mathrm{Al}$ & $\mathrm{Ti}$ & $\mathrm{Co}$ & $\mathrm{Ni}$ & $\mathrm{In}$ & $\mathrm{Pb}$ & $\mathrm{Cd}$ \\
\hline$T_{\mathrm{D}}, \mathrm{K}$ & 390 & 380 & 386 & 345 & 129 & 87 & 120 \\
$\Lambda \cdot 10^{5}, \mathrm{~m}^{2} / \mathrm{s}$ & 4.4 & 4.9 & 4.4 & 4.4. & 4.6 & 4.0 & 5.0
\end{tabular}




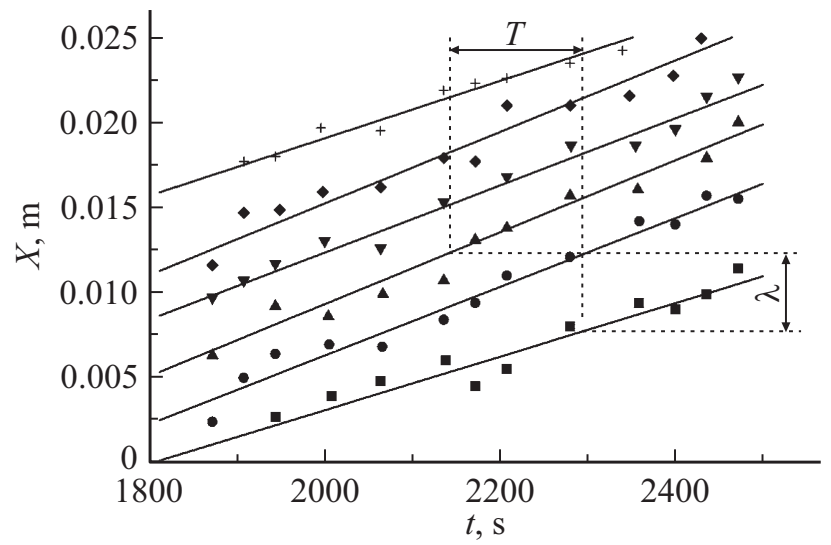

Рис. 1. $X-t$-диаграмма на стадии линейного деформационного упрочнения монокристалла $\mathrm{Cu}$ с ориентацией оси растяжения [139]. Точки разного вида соответствуют различным очагам локальных деформаций вдоль оси образца.

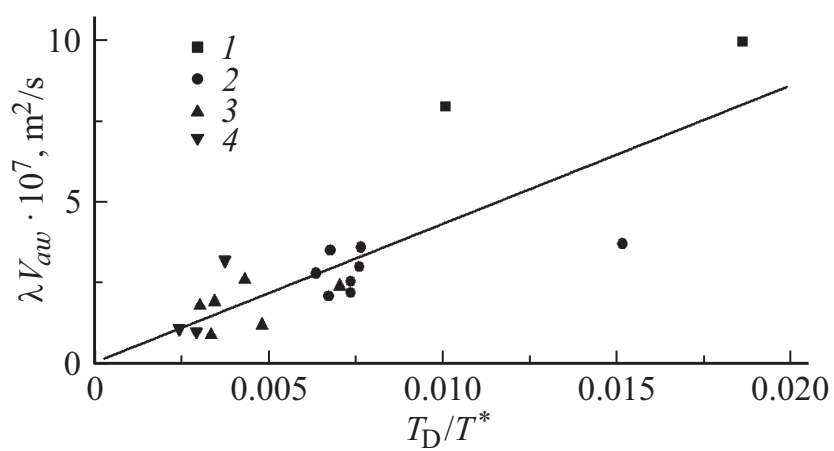

Рис. 2. Зависимость произведения $\lambda V_{a w}$ от величины $T_{\mathrm{D}} / T^{*}$. 1 - элементы третьего периода $(\mathrm{Mg}, \mathrm{Al}), 2$ - элементы четвертого периода ( $\mathrm{Ti}, \mathrm{V}, \alpha$-Fe, $\gamma$-Fe, $\mathrm{Co}, \mathrm{Ni}, \mathrm{Cu}, \mathrm{Zn}$ ), 3 - элементы пятого периода ( $\mathrm{Zr}, \mathrm{Nb}, \mathrm{Mo}, \mathrm{Cd}, \mathrm{In}, \mathrm{Sn}), 4$ элементы шестого периода ( $\mathrm{Hf}, \mathrm{Ta}, \mathrm{Pb})$.

В таком случае из сравнения соотношений (1) и (3) следует, что коэффициент в уравнении (1)

$$
\Lambda=\left(2 \hat{Z} \chi^{2} \frac{k_{\mathrm{B}}}{\hbar} T^{*}\right)=\left(2 \hat{Z} \chi^{2} \frac{E_{b}}{\hbar}\right) .
$$

Это соотношение может быть проверено с помощью непосредственного расчета коэффициента $\Lambda$ для конкретных металлов, для которых известны значения $\chi$ и $T^{*}=E_{b} / k_{\mathrm{B}}$. Результаты расчетов для нескольких металлов представлены в таблице. При этом среднее для всех девятнадцати исследованных металлов значение коэффициента составляет $\langle\Lambda\rangle=(5.86 \pm 2.8) \cdot 10^{-5} \mathrm{~m}^{2} / \mathrm{s}$, что близко к экспериментально полученному его значению в соотношении (1).

Таким образом, экспериментально установленное соотношение (1) и его анализ указывают на взаимную обусловленность кинетической характеристики макроскопического процесса развития локализованной пластической деформации $\lambda V_{a w}$, стоящей в левой части уравнения (3), и величин, характеризующих свойства кристаллической решетки деформируемого вещества. Как известно [5], параметр Дебая характеризует энергию колебаний кристаллической решетки твердого тела. Кроме того, величины, входящие в коэффициент пропорциональности в правой части уравнения (3), также связаны с типичными решеточными свойствами деформируемой среды. Заметим, что появление постоянных Больцмана и Планка в уравнении (3) косвенно подтверждает возможность использования квантовых представлений при описании пластичности $[1,6,7]$.

\section{Финансирование работы}

Работа выполнена в рамках Программы фундаментальных научных исследований государственных академий наук на 2013-2020 гг. (направление III.23).

\section{Конфликт интересов}

Авторы заявляют, что у них нет конфликта интересов.

\section{Список литературы}

[1] Зуев Л.Б. Автоволновая пластичность. Локализация и коллективные моды. М.: Физматлит, 2018. 207 с.

[2] Зуев Л.Б., Баранникова С.А., Лунев А.Г. // Успехи физики металлов. 2018. Т. 19. № 4. С. 379-421.

[3] Физико-химические свойства элементов. Справочник / Под ред. Г.В. Самсонова. Киев: Наук. думка, 1965. 807 с.

[4] Зуев Л.Б. // Металлофизика и новейшие технологии. 2016. T. 38. № 10. C. 1335-1349.

[5] Newnham R.E. Properties of materials. Oxford: University Press, 2005. $378 \mathrm{p}$.

[6] Billigsley J.P. // Int. J. Solids Struct. 2001. V. 38. N 12. P. 42214234.

[7] Zuev L.B., Barannikova S.A. // Solid State Phenom. 2011. V. 172-174. P. 1279-1283. 